Cardiopulmonary

Support and

Physiology

\title{
Multimodal evaluation of in vivo magnetic resonance imaging of myocardial restoration by mouse embryonic stem cells
}

\author{
Stephen L. Hendry, II, MD, ${ }^{a *}$ Koen E. A. van der Bogt, MS, ${ }^{a, e *}$ Ahmad Y. Sheikh, MD, ${ }^{a}$ Takayasu Arai, MD, \\ Scott J. Dylla, PhD, ${ }^{\mathrm{c}}$ Micha Drukker, PhD, ${ }^{\mathrm{c}}$ Michael V. McConnell, MD, ${ }^{\mathrm{b}}$ Ingo Kutschka, MD, ${ }^{\mathrm{a}}$ Grant Hoyt, BS, \\ Feng Cao, MD, PhD, ${ }^{d}$ Irving L. Weissman, MD, ${ }^{c}$ Andrew J. Connolly, MD, PhD, ${ }^{c}$ Marc P. Pelletier, MD, ${ }^{a}$ \\ Joseph C. Wu, MD, PhD, ${ }^{b, d}$ Robert C. Robbins, MD, and Phillip C. Yang, MD ${ }^{b}$
}

From the Department of Cardiothoracic Surgery, ${ }^{\mathrm{a}}$ the Division of Cardiovascular Medicine, ${ }^{\mathrm{b}}$ and the Department of Pathology, ${ }^{\mathrm{c}}$ Stanford University School of Medicine, and the Molecular Imaging Program at Stanford (MIPS), ${ }^{\mathrm{d}}$ Stanford University, Stanford, Calif; and the Department of Surgery, Leiden University Medical Center, Leiden, The Netherlands. ${ }^{\mathrm{e}}$

Supported by the NRSA Fellowship HL082447-01 (S.L.H.), Donald W. Reynolds Foundation (P.C.Y.), Falk Cardiovascular Research Fund (R.C.R.), and National Institutes of Health grants F32 and K23 HL04338-01 (P.C.Y.).

* Both authors contributed equally to this study.

Received for publication Sept 4, 2007; revisions received Dec 5, 2007; accepted for publication Dec 18, 2007.

Address for reprints: Phillip C. Yang, MD, Department of Cardiovascular Medicine, Falk Cardiovascular Research Center, Stanford University Medical Center, 300 Pasteur Dr, Stanford, CA 94305 (E-mail: pyang@ cvmed.stanford.edu)

J Thorac Cardiovasc Surg 2008;136:1028 37

$0022-5223 / \$ 34.00$

Copyright $\odot 2008$ by The American Association for Thoracic Surgery

doi:10.1016/j.jtcvs.2007.12.053
Objective: Mouse embryonic stem cells have demonstrated potential to restore infarcted myocardium after acute myocardial infarction. Although the underlying mechanism remains controversial, magnetic resonance imaging has provided reliable in vivo assessment of functional recovery after cellular transplants. Multimodal comparison of the restorative effects of mouse embryonic stem cells and mouse embryonic fibroblasts was performed to validate magnetic resonance imaging data and provide mechanistic insight.

Methods: SCID-beige mice $(n=55)$ underwent coronary artery ligation followed by injection of $2.5 \times 10^{5}$ mouse embryonic stem cells, $2.5 \times 10^{5}$ mouse embryonic fibroblasts, or normal saline solution. In vivo magnetic resonance imaging of myocardial restoration by mouse embryonic stem cells was evaluated by (1) in vivo pressurevolume loops, (2) in vivo bioluminescence imaging, and (3) ex vivo TaqMan (Roche Molecular Diagnostics, Pleasanton, Calif) polymerase chain reaction and immunohistologic examination.

Results: In vivo magnetic resonance imaging demonstrated significant improvement in left ventricular ejection fraction at 1 week in the mouse embryonic stem cell group. This finding was validated with (1) pressure-volume loop analysis demonstrating significantly improved systolic and diastolic functions, (2) bioluminescence imaging and polymerase chain reaction showing superior posttransplant survival of mouse embryonic stem cells, (3) immunohistologic identification of cardiac phenotype within engrafted mouse embryonic stem cells, and (4) polymerase chain reaction measuring increased expressions of angiogenic and antiapoptotic genes and decreased expressions of antifibrotic genes.

Conclusion: This study validates in vivo magnetic resonance imaging as an effective means of evaluating the restorative potential of mouse embryonic stem cells.

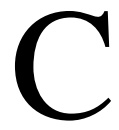
ellular therapy is rapidly emerging as a potential therapeutic option after acute myocardial infarction (AMI). ${ }^{1}$ Although studies have shown that transplants of stem cells derived from different lineages can provide significant functional recovery in the setting of AMI, the exact mechanisms of cell-mediated restoration have not been established. ${ }^{2}$ There are several theories regarding the possible 


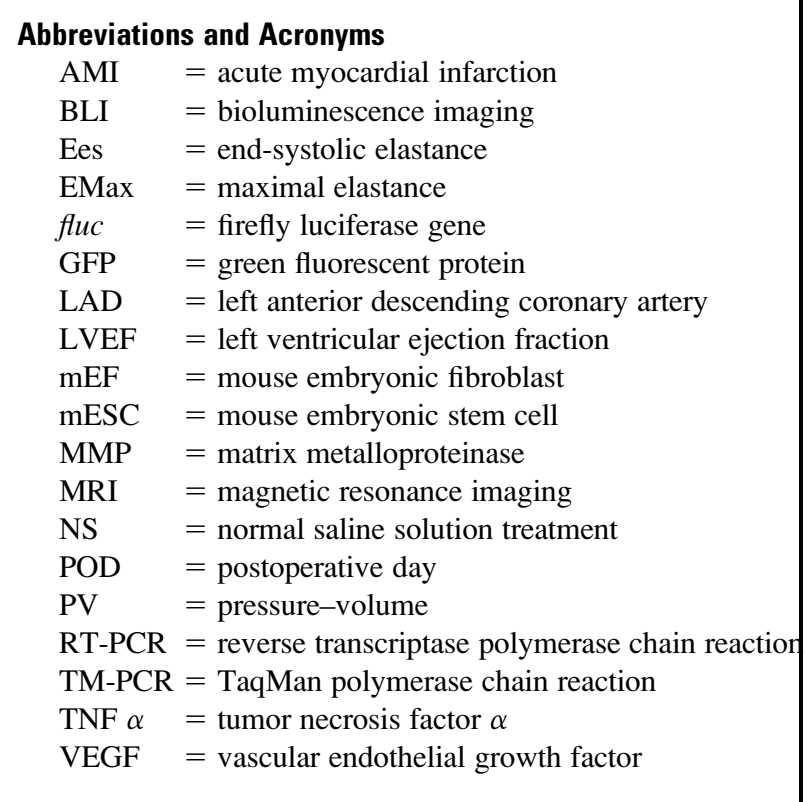

mechanisms underlying myocardial restoration after cellular therapy: (1) augmentation of the infarct region's elasticity, preserving regional systolic and diastolic functions; (2) contractility of engrafted cells, improving systolic function; (3) angiogenesis, enhancing regional myocardial perfusion; and (4) paracrine effects, modulating the progression of cardiac remodeling. ${ }^{3-6}$

In vivo magnetic resonance imaging (MRI) has demonstrated improved cardiac function after stem cell transplants in both preclinical and clinical investigations. ${ }^{1,7-11}$ These findings have led to questions regarding the validity of such data, and more importantly regarding the potential mechanisms underlying myocardial restoration. This investigation addresses these issues by validating in vivo MRI evaluation of myocardial restoration through a systematic comparison of the restorative potential of "nonspecific" mouse embryonic fibroblasts (mEFs) with the more biologically active, self-renewing, pluripotent mouse embryonic stem cells (mESCs) in a murine model of AMI. This is the first study to validate in vivo MRI at a functional level and to conduct a multimodal evaluation of physiologic, cellular, and molecular mechanisms of mESC-mediated myocardial restoration.

\section{Materials and Methods Cell Culture}

Undifferentiated D3-derived mESCs (American Type Culture Collection, Manassas, Va) were cultured in Dulbecco modified Eagle medium (Invitrogen Corporation, Carlsbad, Calif) with $15 \%$ fetal calf serum (HyClone Laboratories Inc, Logan, Utah), 100-mg/mL penicillin-streptomycin, 1-mmol/L sodium pyruvate, 2-mmol/L L-glutamine, 0.1-mmol/L nonessential amino acids, 0.1-mmol/L $\beta$-mercaptoethanol (Invitrogen), and $106-\mu / \mathrm{mL}$ leukemia inhibitory factor (Chemicon International, Inc, Temecula, Calif). Fresh mEFs were prepared from 13-day embryos, which were minced and passed 10 times through a 21-gauge needle. Cells were seeded in $10-\mathrm{cm}$ culture dishes and were propagated for two passages in Dulbecco modified Eagle medium with $10 \%$ fetal calf serum and $100-\mathrm{mg} / \mathrm{mL}$ penicillin-streptomycin solution. Both mESCs and mEFs were transfected with a lentiviral vector carrying a cytomegalovirus promoter driving both a firefly luciferase $(f l u c)$ reporter gene and the gene for green fluorescent protein $(G F P)$. Cells underwent fluorescence-activated cell sorting for GFP and single clone selection; the clone was adapted to feeder-free conditions. Before injection, the cells were trypsinized $(0.25 \%$ trypsin $/ 0.02 \%$ ethylenediaminetetraacetic acid) and washed with Dulbecco modified Eagle medium containing $10 \%$ serum. After centrifugation, the cells were washed with phosphate-buffered saline solution, centrifuged, and resuspended in phosphate-buffered saline solution for injection 1 hour after trypsinization.

\section{Experimental Animals}

Animal care and interventions were provided in accordance with the Laboratory Animal Welfare Act, and all animals received humane care and treatment in accordance with the "Guide for the Care and Use of Laboratory Animals" (www.nap.edu/catalog/5140.html). Immunotolerant SCID-beige female mice (8-12 weeks old; Charles River Laboratories, Inc, Wilmington, Mass) were anesthetized in an isoflurane inhalational chamber and endotracheally intubated with a 20-gauge angiocatheter (Ethicon Endo-Surgery, Inc, Cincinnati, Ohio). Ventilation was maintained with a Harvard rodent ventilator (Harvard Apparatus, Inc, Holliston, Mass). AMI was created by ligation of the mid left anterior descending coronary artery (LAD) through a left thoracotomy. The mice were randomly allocated to three groups: (1) LAD ligation with normal saline solution injection (NS group, $\mathrm{n}=10$ ), (2) LAD ligation with mESC injection (mESC group, $n=25$ ), and (3) LAD ligation with $\mathrm{mEF}$ injection ( $\mathrm{mEF}$ group, $\mathrm{n}=20$ ). The infarct region was injected with a Hamilton syringe containing a $25-\mathrm{mL}$ volume of $250,000 \mathrm{mESCs}, 250,000$ mEFs, or normal saline solution. Cell suspensions contained rhodamine beads $\left(6 \times 10^{5}\right)$ to ensure injection accuracy. After chest tube placement, the chest was closed in two layers with 5-0 Vicryl suture (Ethicon, Inc, Somerville, NJ).

\section{In Vivo MRI}

One week after transplant, cardiac MRI was performed with a Unity Inova console (Varian, Inc, Palo Alto, Calif) controlling a 4.7-T, 15-cm horizontal-bore magnet (Oxford Instruments, Ltd, Oxford, UK) with GE Techron Gradients (12 G/cm; GE Medical Systems, Milwaukee, Wis) and a volume coil with a diameter of $3.5 \mathrm{~cm}$ (Varian). The electrocardiographic gating was optimized with two subcutaneous precordial leads with respiratory motion and body temperature monitors (SA Instruments, Inc, Stony Brook, NY). Left ventricular function was evaluated with electrocardiographically triggered cine sequence (echo-time $2.8 \mathrm{~ms}$, repetition time $160 \mathrm{~ms}$, flip angle $60^{\circ}$, field of view $3.0 \mathrm{~cm}^{2}$, matrix $128 \times 128$, slice gap $0 \mathrm{~mm}$, slice thickness $1.0 \mathrm{~mm}$, number of excitations 8 , and 12 cardiac phases). The imaging plane was localized with double-oblique acquisition. The data were analyzed with MRVision software (MRVision Co, Winchester, Mass). Left ventricular ejection fraction (LVEF) and left ventricular end-diastolic and end-systolic volumes 
were calculated by tracing the endocardial borders in end-systole and end-diastole.

\section{Pressure-Volume Loop Analysis}

One week after transplant, ventricular performance was assessed by pressure-volume (PV) loop analysis with a $1.4 \mathrm{~F}$ conductance catheter (Millar Instruments, Inc, Houston, Tex) before the animals were sacrificed. The closed-chest technique was used; this consisted of a midline neck incision to access the left external jugular vein with PE10 tubing (Intramedic; BD Diagnostic Systems, Sparks, Md). The right carotid artery was cannulated with the Millar catheter, which was advanced through the aortic valve into the left ventricle. The PV relations were measured at baseline and during inferior vena caval occlusion. The measurements of segmental conductance were recorded, which allowed extrapolation of the left ventricular volume. When coupled with pressure, the generation of ventricular PV relationships allowed precise hemodynamic characterization of ventricular systolic and diastolic function and loading conditions. ${ }^{12}$ These data were analyzed with PVAN 3.4 Software (Millar) and Chart/Scope software (ADInstruments, Inc, Colorado Springs, Colo).

\section{In Vitro $f$ luc expression Assay}

On the day of operation, parallel sets of cells from the same plates as the injected cells were trypsinized, resuspended in phosphate-buffered saline solution, and divided into a 6-well plate in different concentrations. After administration of D-luciferin $(4.5 \mu \mathrm{g} / \mathrm{mL}$; Caliper Life Sciences/Xenogen, Alameda, Calif), peak signal expressed as photons per second per square centimeter per steridian was measured with a charge-coupled device camera (Xenogen).

\section{In Vivo Optical Bioluminescence Imaging}

Optical bioluminescence imaging (BLI) was performed with $8 \times 5$ minute acquisition scans on a charge-coupled device camera (IVIS 50; Xenogen). Recipient mice were anesthetized and placed in the imaging chamber. After acquisition of a baseline image, mice were intraperitoneally injected with D-luciferin $(400 \mathrm{mg} / \mathrm{kg}$ body weight; Xenogen). Peak signal from a fixed region of interest was evaluated with Living Image 2.50 software (Xenogen).

\section{Ex Vivo TaqMan Polymerase Chain Reaction for Cell Survival and Expressions of Genes of Interest}

Because the transplanted cells were derived from male mice and were transplanted into female recipients, the surviving mESCs in the explanted hearts could be quantified by using TaqMan (Roche Molecular Diagnostics, Pleasanton, Calif) polymerase chain reaction (TM-PCR) to track the Sry locus found on the Y chromosome. Whole explanted hearts were minced and homogenized in DNAzol (Invitrogen). RNA was extracted from the myocardium after treatment with TRIzol reagent (Invitrogen). TM-PCR was performed with the SuperScript II reverse transcriptase PCR (RT-PCR) kit (Invitrogen). To assess the expressions of several genes of interest, relative quantitation of mouse primers was performed for the following: matrix metalloproteinases (MMPs) $1 \beta, 2$, 9, and 14; tumor necrosis factor $\alpha$ (TNF- $\alpha)$, vascular endothelial growth factor $\mathrm{A}$ (VEGF-A), procollagen $2 \alpha 1$, transforming growth factor $\beta$, angiotensin-converting enzyme, insulinlike growth factor 1 , kinase insert domain protein receptor, and nuclear factor $\kappa \mathrm{B}-1$ (Applied Biosystems, Foster City, Calif). The fluorogenic probes contained a 5'-FAM report dye and 3'-BHQ1 quencher dye. TaqMan 18S Ribosomal RNA (Applied Biosystems) was used as a control gene. RT-PCR reactions were conducted in iCycler IQ Real-Time Detection Systems (Bio-Rad Laboratories Inc, Hercules, Calif).

\section{Histologic Analysis}

Hearts were flushed with normal saline solution and subsequently placed in $2 \%$ paraformaldehyde for 2 hours at room temperature, followed by 12 to 24 hours in $30 \%$ sucrose at $4^{\circ} \mathrm{C}$. The tissue was embedded in Optical Cutting Temperature Compound (TissueTek; Sakura Finetek USA Inc, Torrance, Calif) and snap frozen on dry ice. Sections of $5 \mu \mathrm{m}$ were cut in both the proximal and apical regions of the infarct zone. Slides were stained with hematoxylin and eosin, anti-GFP (anti-green fluorescent protein, rabbit IgG fraction, anti-GFP Alexa Fluor 488 conjugate, 1:200; Molecular Probes, Inc, Eugene, Ore), anti-troponin I (H-170 rabbit polyclonal IgG for cardiac muscle, 1:100; Santa Cruz Biotechnology, Inc, Santa Cruz, Calif), and anti-connexin 43 (rabbit polyclonal, 1:100; Sigma, St Louis, Mo). Stained tissue was examined with a Leica DMRB fluorescent microscope (Leica Microsystems Inc, Deerfield, Ill) and a Zeiss LSM 510 2-photon confocal laser scanning microscope (Carl Zeiss, Inc, Thornwood, NY). Cell engraftment was confirmed by identification of GFP expression under fluorescent microscopy. Colocalization of troponin, $\alpha$-sarcomeric actin, and connexin 43 with GFP was visualized with streptavidin conjugated with Alexa Fluor Red 555 (Invitrogen).

\section{Statistical Analysis}

Descriptive statistics included mean and SE. Comparison between groups was performed with the Student $t$ test for independent and normally distributed data variables with SPSS 11.0 (SPSS Inc, Chicago, Ill). For comparisons between multiple groups, analysis of variance with Bonferroni correction was used.

\section{Results}

\section{Quantitation of LVEF by in Vivo MRI}

As seen in Figure 1 ( $A$ and $B$ ), MRI indicated that AMI led to significant reduction in $\mathrm{LVEF}$ at 1 week in all groups relative to sham-operated, normal hearts $(60.9 \% \pm 1.4 \%, \mathrm{n}=5, P<$ $.01)$, illustrating the effectiveness of this murine AMI model. In the mESC group, a significant improvement of LVEF $(40.2 \% \pm 2.0 \%, \mathrm{n}=23)$ was seen relative to both $\mathrm{mEF}$ $(29.4 \% \pm 1.5 \%, \mathrm{n}=17)$ and NS $(26.4 \% \pm 1.8 \%, \mathrm{n}=6$, $P<.05)$ groups. No significant improvement was observed in the $\mathrm{mEF}$ group relative to the NS group. Measurements of left ventricular end-diastolic and end-systolic volumes are included in Table E1.

\section{Measurement of Maximal Elastance and End-Systolic Elastance by PV Loop Analysis}

As seen in Figure 1, $C$, PV loop analysis demonstrated significantly compromised maximal elastance (Emax) in the $\mathrm{mEF}$ group $(9.95 \pm 1.4 \mathrm{~mm} \mathrm{Hg} / \mathrm{mL}, \mathrm{n}=4)$ and the NS group $(5.8 \pm 1.2 \mathrm{~mm} \mathrm{Hg} / \mathrm{mL}, \mathrm{n}=4)$ relative to that in normal hearts $(22.6 \pm 2.5 \mathrm{~mm} \mathrm{Hg} / \mathrm{mL}, \mathrm{n}=5, P<.05)$ at 1 week; however, Emax was noticeably preserved in the mESC group (18.4 \pm 


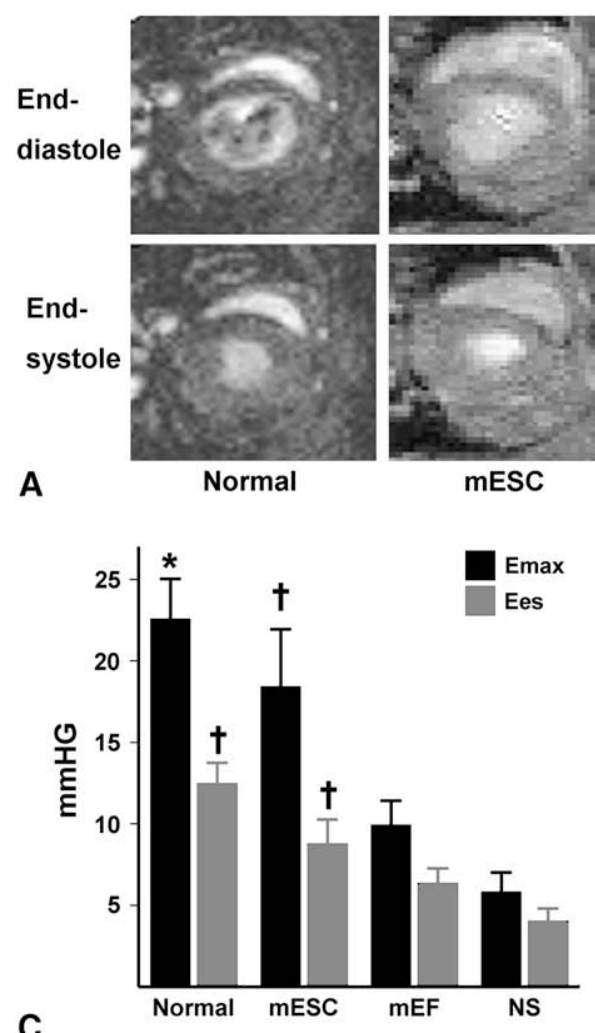

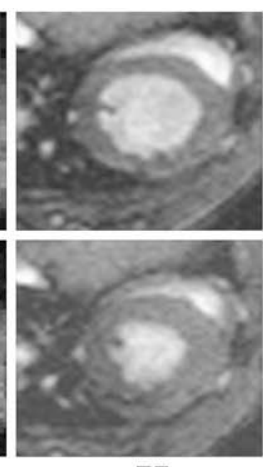

mEF

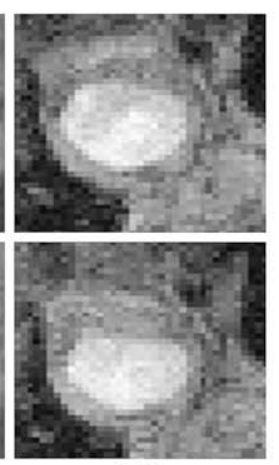

NS
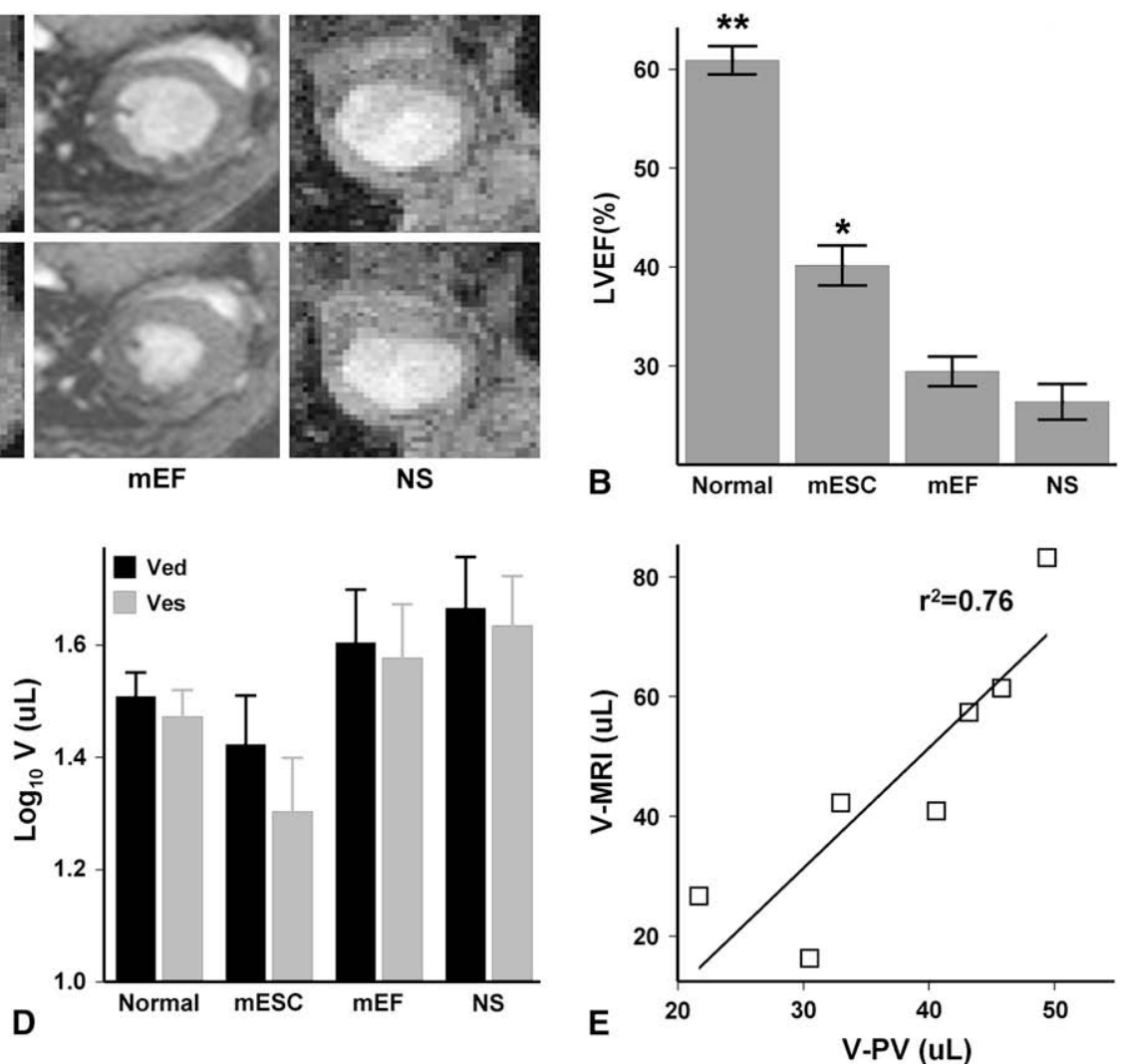

Figure 1. Functional outcomes after mouse embryonic stem cell and mouse embryonic fibroblast transplants. $A$, Representative magnetic resonance images for each group (normal, mouse embryonic stem cell [mESC], mouse embryonic fibroblast [mEF], and normal saline-treated [NS] shown in end-diastole and end-systole at 1 week after left anterior descending coronary artery ligation and cellular transplant. Mouse embryonic stem cell group demonstrated increased left ventricular ejection fraction, with visual confirmation from magnetic resonance images. B, One week after left anterior descending coronary artery ligation and cellular transplant, magnetic resonance imaging indicated significant improvement in left ventricular ejection fraction (LVEF) in mouse embryonic stem cell (mESC) group versus mouse embryonic fibroblast (mEF) and normal saline-treated (NS) groups. Asterisk represents $P<.05$ versus mouse embryonic fibroblast and normal saline-treated groups by analysis of variance. Double asterisk indicates $\boldsymbol{P}<.01$ versus all groups by analysis of variance. C, One week after left anterior descending coronary artery ligation and cellular transplant, PV loop analysis demonstrated compromised maximum elastance (Emax) in mouse embryonic fibroblast (mEF) and normal saline-treated (NS) groups relative to normal hearts. Preserved maximum elastance was noticeable in mouse embryonic stem cell (mESC) group, with significantly higher value than in normal-saline treated group. No significant improvement was observed in maximal elastance in mouse embryonic fibroblast group relative to normal saline-treated group. End-systolic elastance (Ees) data show significant decrease in normal saline-treated (NS) group relative to normal hearts and significant preservation of end-systolic elastance in mouse embryonic stem cell (mESC) group relative to normal saline-treated group. No significant improvement was observed in mouse embryonic fibroblast (mEF) group relative to normal saline-treated group. Asterisk indicates $p<.05$ versus mouse embryonic fibroblast and normal saline-treated groups. Dagger indicates $P<.05$ versus normal saline-treated group ( $n>4$ / group by analysis of variance). D, Pressure-volume loop measurements of left ventricular volumes (V) in end-systole (Ves) and end-diastole (Ved) show nonsignificantly decreased ventricular dilatation in mouse embryonic stem cell (mESC) group relative to other treatment groups. mEF, mouse embryonic fibroblast; NS, normal saline treatment. E, Scatter plot of average left ventricular volumes in each group measured by pressure-volume loop (V-PV) and magnetic resonance imaging $(V-M R I)$, with $r^{2}=0.76$. 


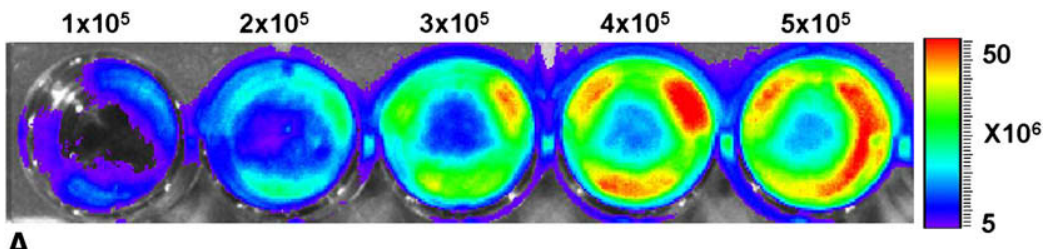

A

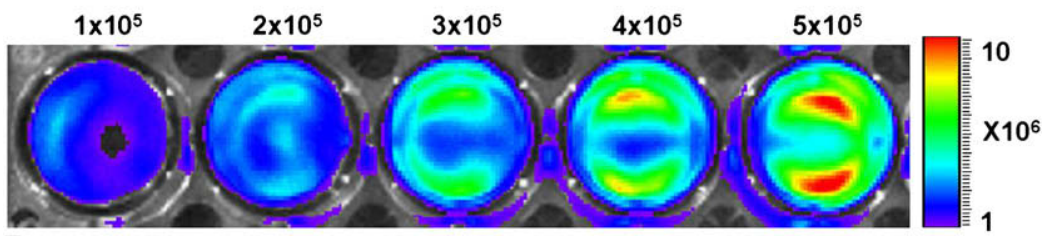

B

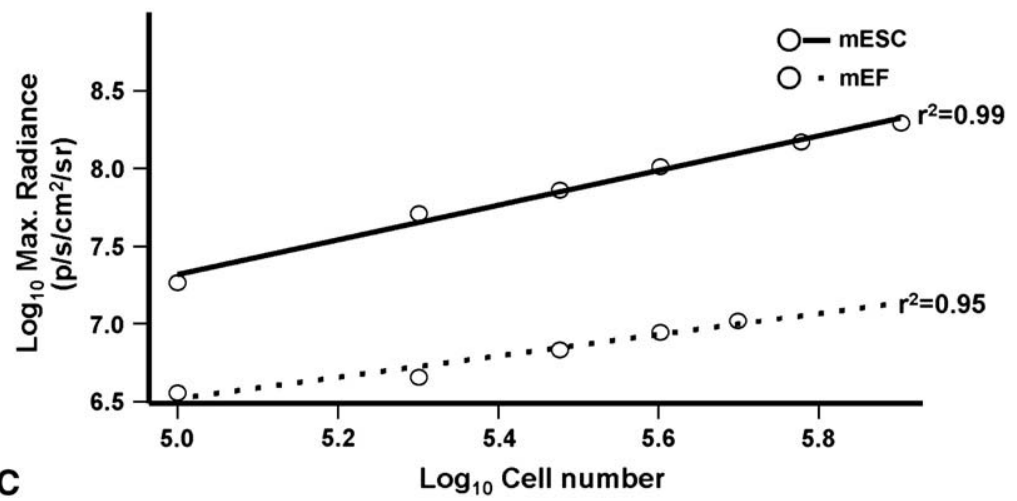

Figure 2. In vitro firefly luciferase signal correlates with cell number. A and B, Bioluminescence images show increasing signal with increasing cell number in mouse embryonic stem cells $(A)$ and mouse embryonic fibroblasts (B). Bars represent maximum radiance. C, Correlation plot shows robust correlation between signal and cell number for mouse embryonic stem cells (mESC, $\left(r^{2}=0.99\right)$ and mouse embryonic fibroblasts $\left(m E F, r^{2}=0.95\right)$.
$3.5 \mathrm{~mm} \mathrm{Hg} / \mathrm{mL}, \mathrm{n}=4$ ) and significantly higher than that in the mEF and NS groups $(P<.05)$. No significant improvement was observed in Emax in the mEF group relative to the NS group. The end-systolic elastance (Ees) data paralleled these findings, with a significant decrease in the NS group (4.1 $\pm 0.7 \mathrm{~mm} \mathrm{Hg} / \mathrm{mL}, \mathrm{n}=4)$ relative to normal hearts $(12.5 \pm$ $1.2 \mathrm{~mm} \mathrm{Hg} / \mathrm{mL}, \mathrm{n}=5, P<.05)$ and a significant preservation of Ees in the mESC group $(8.8 \pm 1.5 \mathrm{~mm} \mathrm{Hg} / \mathrm{mL}, \mathrm{n}=4)$ relative to the $\mathrm{mEF}$ and NS groups $(P<.05)$. No significant improvement in Ees was observed in the mEF group relative to the NS group. As shown in Figure 1, D, the left ventricular volumes measured by PV loop analysis in the $\mathrm{mEF}$ and NS groups demonstrated negative remodeling, whereas hearts in the $\mathrm{mESC}$ group showed the least dilatation. The improved systolic and diastolic functions measured by the PV loops provide physiologic confirmation of in vivo MRI data, with correlation of left ventricular volumes measured by MRI and PV loop (Figure 1,E). Table E1 lists further results of invasive hemodynamic measurements.

\section{Determination of Transplanted Cell Survival by in Vivo BLI and ex Vivo TM-PCR}

Stable mESC and mEF transfection with GFP and $f l u c$ generated mESC-GFP ${ }^{+}-f l u c^{+}$and $\mathrm{mEF}-G F P^{+}-f l u c^{+}$cell lines.
The cells were selected and tested for $f$ luc signal by BLI. For both lines, expression of $f l u c$ signal correlated robustly with cell number $\left(r^{2}=0.99\right.$ and $r^{2}=0.95$, respectively, Figure 2, $A-C)$. BLI was thus validated as a tool for monitoring cell viability quantitatively, with the signal intensity reflecting the number of viable cells in vitro. After transplant of the transfected cells, BLI signal from the mESC group decreased until postoperative day (POD) 2. At PODs 8 and 14 , there was a significant $(P<.01)$ increase in signal as a result of the rapid division of the undifferentiated mESCs (Figure 3, $A$ and $B$ ), probably commencing teratoma formation as already shown in our earlier work. ${ }^{13}$ In the $\mathrm{mEF}$ group, however, signal increased until POD 2 but decreased thereafter, suggesting cell death (Figure $3, A$ and $B$ ). Ex vivo TM-PCR results indicated significantly lower cycle numbers with time in the mESC group relative to the increasing cycle numbers in the $\mathrm{mEF}$ group (Figure 3,C), which is representative of higher number of viable male donor cells in the mESC group. ${ }^{14}$ Ex vivo TM-PCR thus correlated well with the BLI results (Figure 3,D). This finding supports in vivo MRI data in which cell survival, a major biologic property, is significantly enhanced for the transplanted mESCs to remain biologically active to restore the injured myocardium. 


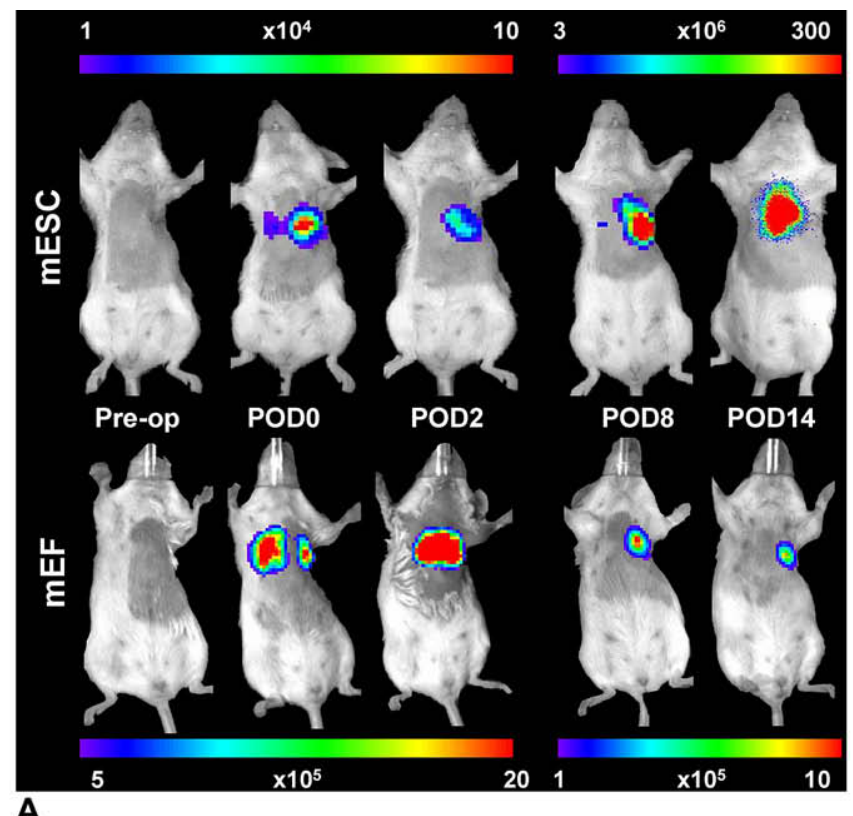

A
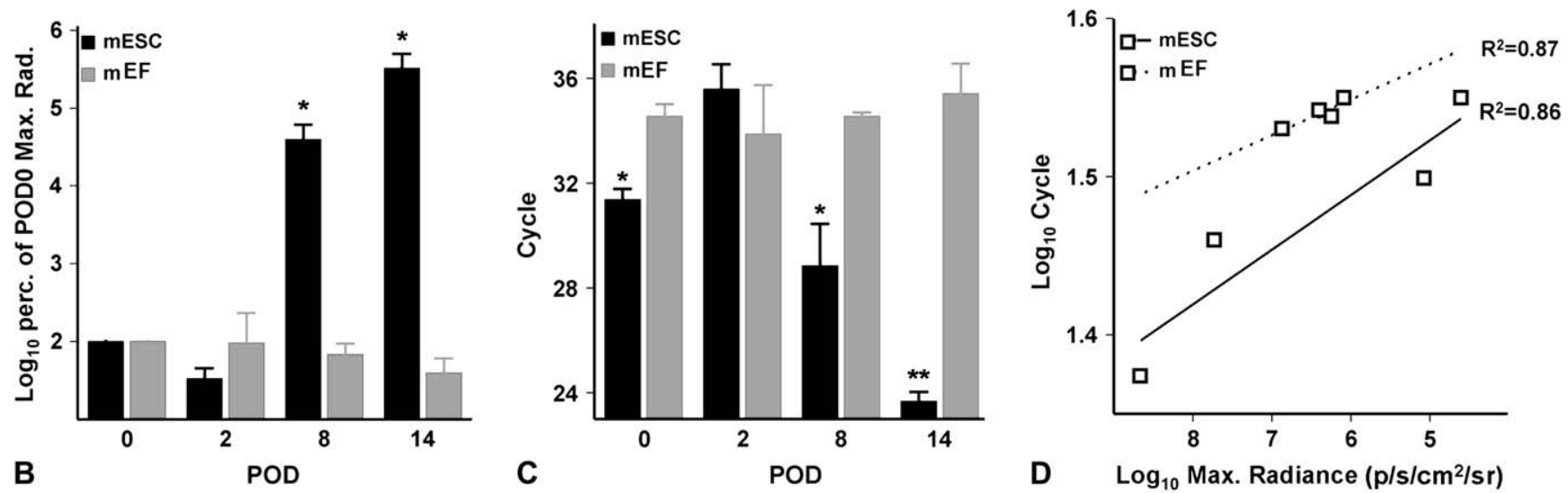

Figure 3. Transplanted cell survival by in vivo bioluminescence and ex vivo TaqMan polymerase chain reaction. $A$, Representative bioluminescence images of mouse embryonic stem cell (mESC) and mouse embryonic fibroblast (mEF) survivals, tracked longitudinally at postoperative days (POD) 0, 2, 8, and 14. Mouse embryonic stem cell survival decreased from postoperative day 0 to postoperative day 2, after which there were notable increases at postoperative days 8 and 14 from rapid division. Color bars represent maximum radiance. B, Normalized plot indicating significant $(P<.01)$ mouse embryonic stem cell ( $m E S C)$ proliferation starting on postoperative day (POD) 2 and gradual cell death in mouse embryonic fibroblast (mEF) group starting on postoperative day 2 (n $>4 /$ group). Ordinate shows $\log _{10}$ percentage of average bioluminescence signal on postoperative day 0 . Asterisk indicates $P<.01$ by analysis of variance versus mouse embryonic stem cells on postoperative days 0 and 2 and versus mouse embryonic fibroblasts for all time points. C, Ex vivo TaqMan polymerase chain reaction for Sry gene representing male mouse embryonic stem cell (mESC) survivals at postoperative days (PODs) $0,2,8$, and 14, demonstrating similar trend to that of in vivo bioluminescence imaging (n $>4 /$ group). Asterisk indicates $P<.05$ versus mouse embryonic fibroblasts by analysis of variance. Double asterisk indicates $P<.01$ versus mouse embryonic fibroblasts (mEF) by analysis of variance. $D$, Correlation plot showing correlations $\left(r^{2}=0.86\right.$ and $r^{2}=0.87$ for mouse embryonic stem cell (mESC) and mouse embryonic fibroblast (mEF) groups, respectively) between in vivo bioluminescence imaging and ex vivo TaqMan polymerase chain reaction. 
Gene expression profiling by TM-PCR for MMP-1 $\beta$, MMP-2, MMP-9, MMP-14, TNF- $\alpha$, VEGF-A, procollagen $2 \alpha 1$, insulinlike growth factor 1 , transforming growth factor- $\beta$, angiotensin-converting enzyme, kinase insert domain protein receptor, and nuclear factor $\kappa \mathrm{B}-1$

Relative quantitation of messenger RNA expression of 12 genes (4 mice/group) with RT-PCR demonstrated significant increase of TNF- $\alpha$ in the mESC group relative to the mEF and NS groups (501\% vs $77 \%$ and $219 \%$, respectively, $P<.01$ ). Significant upregulation of VEGF-A was also observed in the $\mathrm{mESC}$ group relative to the $\mathrm{mEF}$ and NS groups (104\% vs $13 \%$ and $42 \%$, respectively, $P<.01$ ). On the other hand, mESC-treated mice demonstrated a trend toward downregulation of MMP- $1 \beta$ and procollagen $2 \alpha 1$ expressions relative to the NS group $(P>.05)$. The messenger RNA expressions of the remainder of genes (for insulinlike growth factor 1, nuclear factor $\kappa \mathrm{B}-1$, MMP-2, MMP-9, MMP-14, transforming growth factor $\beta$, angiotensin-converting enzyme, and kinase insert domain protein receptor) did not demonstrate significant difference among $\mathrm{mESC}, \mathrm{mEF}$, and NS groups. The gene expression profiles of TNF- $\alpha$, which may have had an enhanced antiapoptotic effect, and VEGF-A, consistent with proangiogenic effects, were demonstrated in the mESC group (Figure 4).

\section{Immunohistologic Evidence of mESC Cardiomyocyte Differentiation}

As seen in Figure $5(A-D)$, cross-sectional images of hematoxylin and eosin-stained hearts demonstrated thinning of myocardial tissue and dilatation of the left ventricle after LAD ligation. In the mESC group, however, restoration of the left ventricular wall mass and reduction of left ventricular dilatation were seen. Colocalization of GFP with troponin and $\alpha$-sarcomeric actin in isolated cells demonstrated potential differentiation of the mESCs into cardiomyocytes, as shown in Figure $5(E-H)$. Colocalization of GFP with connexin 43 suggests the formation of gap junctions, as represented in Figure 5, I.

\section{Discussion}

Cardiomyocyte death or dysfunction after an AMI results in pathologic remodeling of the left ventricle, with the eventual sequela of heart failure. Despite recent reports of the regenerative potential of cell-based therapies in the injured myocardium, a definitive mechanism of this enhanced myocardial function has not been elucidated. In this study, we have provided independent support for in vivo MRI data by PV loop examination and have established the fundamental biologic importance of cell survival, cell differentiation, and paracrine effects as potential mechanisms of mESCs underlying myocardial restoration. The investigation focused on the immediate effects of cellular transplants after AMI because teratoma formation would likely have interfered with reliable measure-

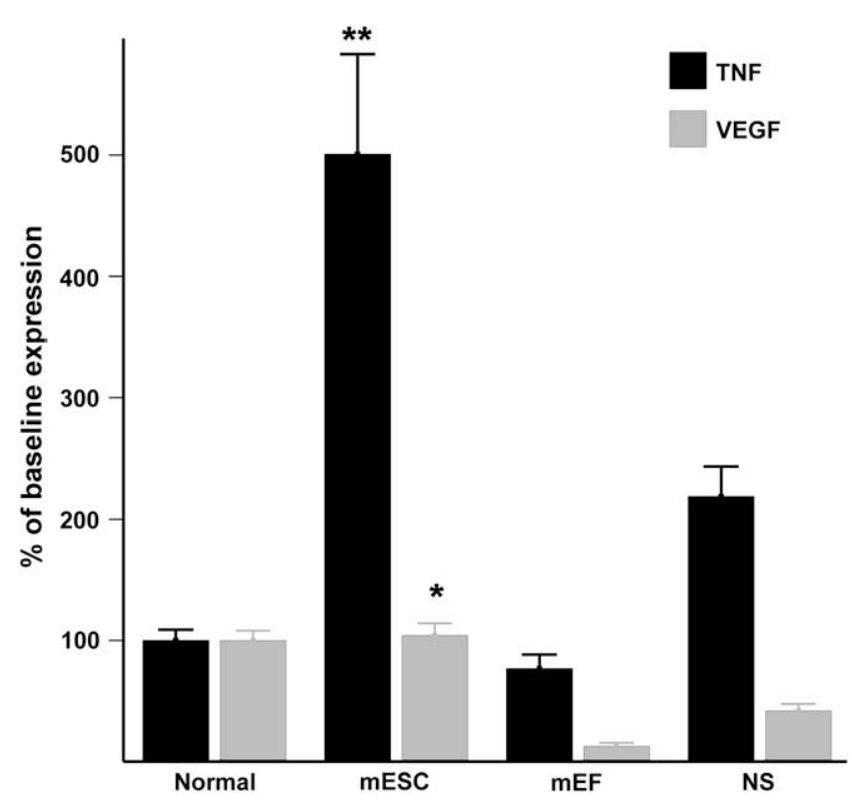

Figure 4. Relative quantitation of tumor necrosis factor $\alpha$ (TNF) and vascular endothelial growth factor A (VEGF) expressions. Relative quantitation of messenger RNA expression on postoperative day 7, normalized as percentage of expression in normal, shamoperated hearts. Significant upregulation is noticeable in mouse embryonic stem cell (mESC) group. Asterisk indicates $P<.01$ by analysis of variance versus mouse embryonic fibroblast (mEF) and normal saline-treated (NS) groups. Double asterisk indicates $\boldsymbol{P}<.01$ by analysis of variance versus all other groups.

ments during longer follow-up, ${ }^{15}$ as the greatly increased mESC BLI signal on POD 14 confirmed. Our data demonstrated significantly improved LVEF measured by in vivo MRI, the most commonly used clinical end point. This finding was confirmed by Ees and EMax values measured by PV loop, indicating improved systolic and diastolic functions as early as 1 week after mESC treatment. The improvement in diastolic function in the mESC group can be attributed to the augmentation of the infarct region's elasticity and recovery in the overall EMax. Moreover, we found a correlation between left ventricular volume measurements by MRI and PV loop.

These findings challenge the current notion that myocardial function is improved irrespective of cell type. ${ }^{16,17}$ The preservation of cardiac performance after cellular transplant cannot be attributed merely to the physical scaffolding effect but also must arise from the biologic properties of the transplanted cells. During myocardial ischemia, the ischemic region develops diastolic and subsequent systolic abnormality. ${ }^{18}$ Studies have confirmed the Frank-Starling relationship, in which contractile function is preserved with reduction in ventricular volume. ${ }^{19}$ In fact, our PV loop results indicate a reduction in ventricular volume in the $\mathrm{mESC}$ group. This reduction involves complex yet fundamental biologic processes. 


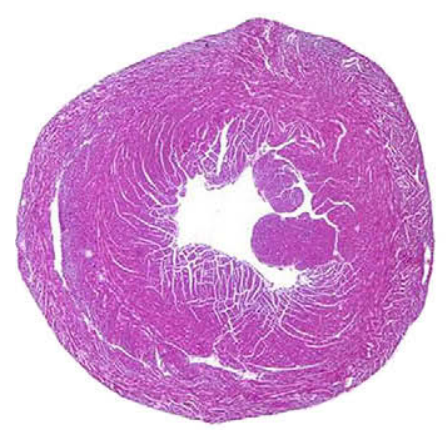

A Normal

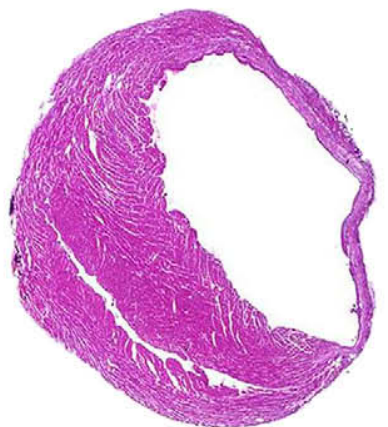

B NS

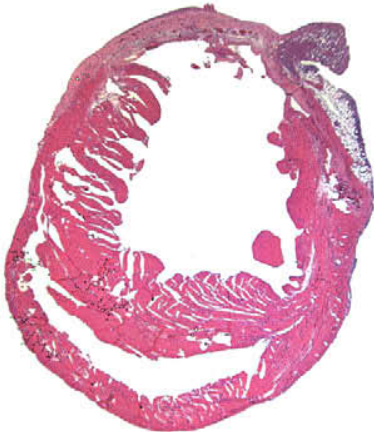

C

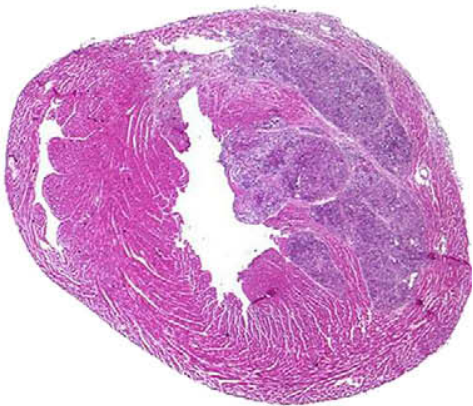

D

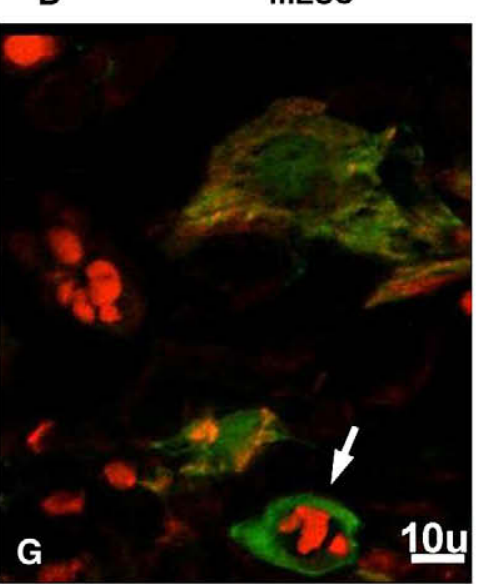

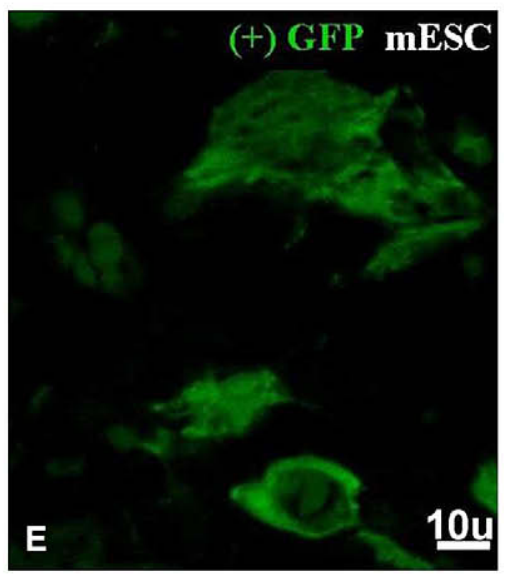
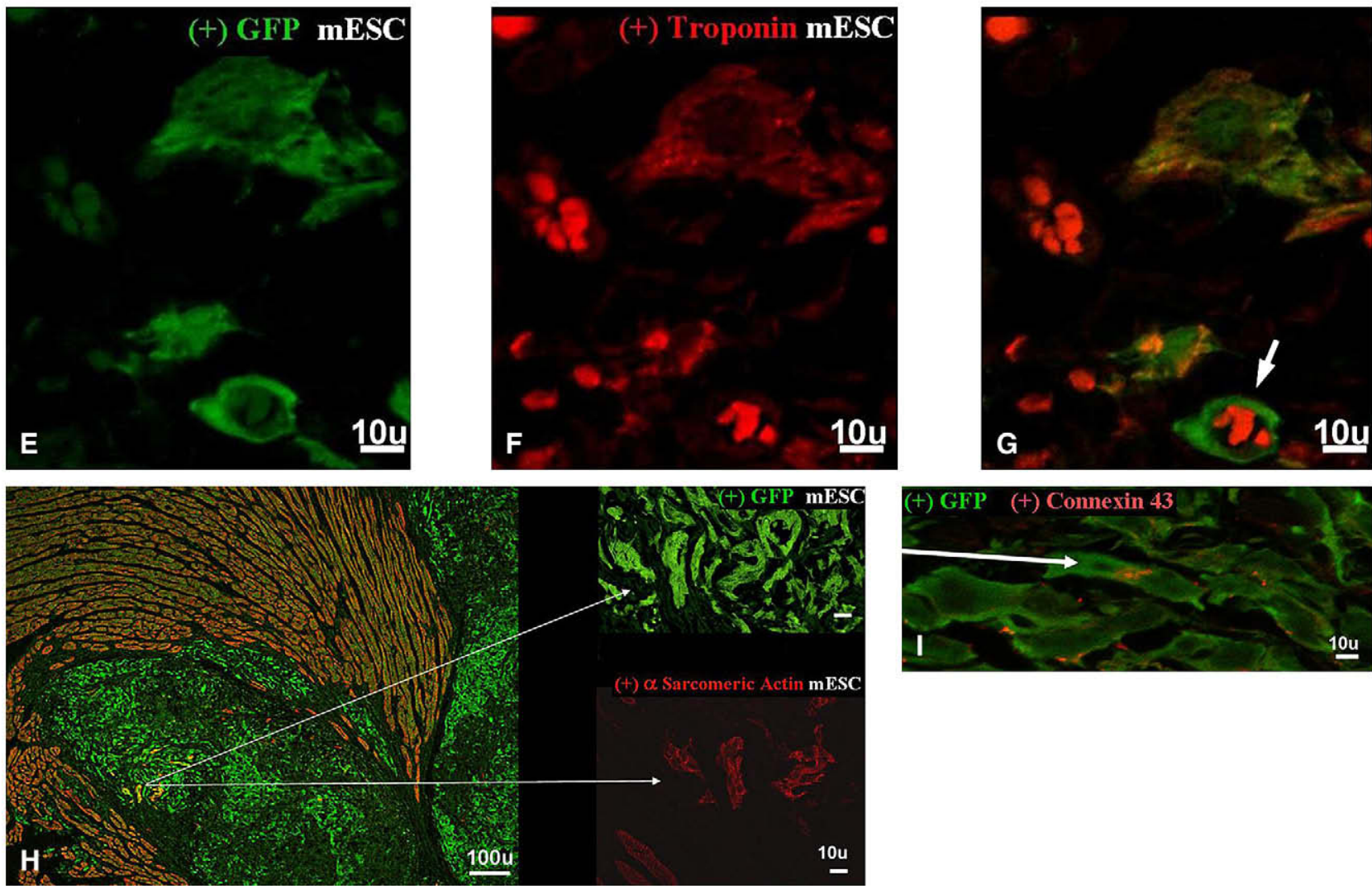

Figure 5. Histopathologic examination of antiremodeling effect and cardiomyocyte differentiation after transplant of mouse embryonic stem cells on postoperative day 7. A-D, Representative cross-sectional images of hematoxylin and eosin-stained normal heart (A); left anterior descending coronary artery ligation control (NS, B), demonstrating thinning and dilatation of ventricular wall; $C$, heart treated with mouse embryonic fibroblasts (mEF); and heart treated with mouse embryonic stem cells (mESC), demonstrating restoration of left ventricular wall mass. $E$, Confocal microscopy showing mouse embryonic stem cells (mESC) staining positive for green fluorescent protein (GFP). Confocal microscopy showing same cells as in $D$ expressing troponin; $7-\mu \mathrm{m}$ clustered round structures probably represent nonspecifically stained red blood cells. G, Overlay of $D$ and $E$, showing colocalization of green fluorescent protein (GFP) with troponin, suggestive of mouse embryonic stem cell-derived cardiomyocyte. Note nonspecifically colored blood cells located inside green fluorescent protein-stained blood vessel (white arrow), suggesting mouse embryonic stem cell-derived neovasculogenesis. $H$, Image showing colocalization of green fluorescent protein (GFP)-positive mouse embryonic stem cells (mESC) with $\alpha$-sarcomeric actin. I, Image showing colocalization of green fluorescent protein (GFP) with connexin 43 (white arrow). 
First, the prolonged survival kinetics of mESCs can offer a longer lasting scaffolding effect, which may help to explain both the reduction in pathologic remodeling and the sustained restoration. More importantly, however, the persistence of viable mESCs generates biologically active stimuli to salvage the injured myocytes. Thus this enhanced survival of mESCs enables a second potential mechanistic explanation for in vivo MRI findings of myocardial restoration, a paracrine effect. The results of ex vivo TM-PCR demonstrate that the potential antiapoptotic cytokine TNF- $\alpha$ was significantly upregulated in the mESC group. TNF- $\alpha$ has been shown to have negative inotropic effects, to induce resistance to hypoxic stress in cardiomyocytes, and to play a role in the recruitment of stem cells. ${ }^{20-23}$ It must be stated, however, that the increased TNF- $\alpha$ expression could also be attributable to a greater mass effect of mESCs, with a subsequent greater endogenous inflammatory reaction, a greater mESC-induced monocyte influx, or teratoma formation leading to endogenous TNF$\alpha$ production. In addition, the observed upregulation of VEGF-A in this study may have promoted angiogenesis to attenuate the ischemic insult and allow recovery of the injured cardiomyocytes. Finally, nonsignificant trends towards downregulation of MMP-1 and procollagen $2 \alpha 1$ have also been detected, which may contribute to decreased cardiac matrix remodeling in failing hearts. ${ }^{24}$ Surprisingly, we did not observe upregulation of MMP-2 and MMP-9. This may have been because of the absence in the SCID mice of T cells, on which MMP-2 and MMP-9 expressions have been shown to depend. ${ }^{25}$ Taken together, these TM-PCR results suggest that multiple mechanisms underlie the restoration by mESC treatment.

The pluripotency of mESCs may lead to regeneration of cardiac tissue after cardiomyogenic differentiation. ${ }^{26}$ To contract synchronously with host cardiomyocytes, newly formed mESC-derived cardiomyocytes must undergo electromechanical coupling through the formation of gap junctions in vivo with the host myocardium. ${ }^{26,27}$ This study provides possible evidence that donor mESCs are capable of engrafting within the host myocardium and differentiating into cardiomyocytes. Although these findings are encouraging, it must be noted that this was a low-frequency event, making it less likely that the observed functional improvement can be attributed to robust cardiac regeneration by mESC-derived cardiomyocytes.

There are several limitations of this study. First, to investigate the acute effect of cell survival, we chose to compare fast-growing, undifferentiated $\mathrm{mESCs}$ with less active mEFs. This gave us the opportunity to study the relationship between cardiac function and cell survival in the relatively short period of 1 week. It must be stated, however, that eventual teratoma formation, as suggested by our BLI findings on POD 14 and consistent with the literature, ${ }^{13,15,28}$ would likely hamper long-term restoration of cardiac function. Second, this study does not provide insight into acute postoperative infarct size, which may have been dependent on cell type.
All operations, however, were conducted by the same experienced microsurgeon who was blinded to the study. Moreover, after 1 week, all operated groups had a significantly compromised LVEF on MRI, leading to the assumption that initial infarct size was comparable among all study groups. Third, we focused on validating the most important MRI data, namely left ventricular end-diastolic and left ventricular end-systolic volumes. Wall thickness, which may have correlated with cell survival, was not measured because we did not anticipate regenerative changes in the immediate period after AMI. Other studies are currently underway to address the regenerative changes by measuring wall thickness.

In conclusion, this is the first study to provide a fundamental functional and biologic evaluation of in vivo MRI in mESC therapy. This study has shown that mESCs are superior to $\mathrm{mEFs}$ in restoring cardiac function in the immediate post-AMI period. A fundamental notion that the cells must at the very least survive to restore the myocardium is confirmed by the finding that the enhanced survival of mESCs is among the key factors in myocardial restoration. Improved survival of transplanted cells may offer not only a physical scaffolding mechanism but, more importantly, biologic support by generating sustained paracrine effects after injury. We have also observed that $\mathrm{mESCs}$ retain the ability to differentiate into cardiomyocytes, albeit at low frequency. Unfortunately, although the pluripotency and robust proliferation are among the major advantages of embryonic stem cells, these characteristics also contribute to teratoma formation, ${ }^{13,15}$ which for the present prevents clinical translation. Further research regarding directed differentiation of mESCs into cardiomyocytes may lead to a safe regenerative therapy for myocardial disease in the future.

We greatly appreciate the assistance with immunohistology from Ms Pauline Chu and the help with PCR from Ms Sally Zhang and Mr Anant Patel.

\section{References}

1. Schachinger V, Erbs S, Elsasser A, Haberbosch W, Hambrecht R, Holschermann $\mathrm{H}$, et al. Intracoronary bone marrow-derived progenitor cells in acute myocardial infarction. N Engl J Med. 2006;355:1210-21.

2. Chien KR. Stem cells: lost in translation. Nature. 2004;428:607-8.

3. Kato S, Spinale FG, Tanaka R, Johnson W, Cooper GT, Zile MR. Inhibition of collagen cross-linking: effects on fibrillar collagen and ventricular diastolic function. Am J Physiol. 1995;269(3 Pt 2):H863-8.

4. Kofidis $T$, de Bruin JL, Yamane T, Tanaka M, Lebl DR, Swijnenburg RJ, et al. Stimulation of paracrine pathways with growth factors enhances embryonic stem cell engraftment and host-specific differentiation in the heart after ischemic myocardial injury. Circulation. 2005;111:2486-93.

5. Li RK, Weisel RD, Mickle DA, Jia ZQ, Kim EJ, Sakai T, et al. Autologous porcine heart cell transplantation improved heart function after a myocardial infarction. J Thorac Cardiovasc Surg. 2000;119:62-8.

6. Tomita S, Li RK, Weisel RD, Mickle DA, Kim EJ, Sakai T, et al. Autologous transplantation of bone marrow cells improves damaged heart function. Circulation. 1999;100(19 Suppl):II247-56.

7. Arai T, Kofidis T, Bulte JW, de Bruin J, Venook RD, Berry GJ, et al. Dual in vivo magnetic resonance evaluation of magnetically labeled 
mouse embryonic stem cells and cardiac function at 1.5 t. Magn Reson Med. 2006;55:203-9.

8. Meyer GP, Wollert KC, Lotz J, Steffens J, Lippolt P, Fichtner S, et al. Intracoronary bone marrow cell transfer after myocardial infarction: eighteen months' follow-up data from the randomized, controlled BOOST (BOne marrOw transfer to enhance ST-elevation infarct regeneration) trial. Circulation. 2006;113:1287-94.

9. Orlic D, Kajstura J, Chimenti S, Jakoniuk I, Anderson SM, Li B, et al. Bone marrow cells regenerate infarcted myocardium. Nature. 2001; 410:701-5.

10. Lunde K, Solheim S, Aakhus S, Arnesen H, Abdelnoor M, Egeland T, et al. Intracoronary injection of mononuclear bone marrow cells in acute myocardial infarction. N Engl J Med. 2006;355:1199-209.

11. Assmus B, Honold J, Schachinger V, Britten MB, Fischer-Rasokat U, Lehmann R, et al. Transcoronary transplantation of progenitor cells after myocardial infarction. $N$ Engl J Med. 2006;355:1222-32.

12. Kass DA, Midei M, Graves W, Brinker JA, Maughan WL. Use of a conductance (volume) catheter and transient inferior vena caval occlusion for rapid determination of pressure-volume relationships in man. Cathet Cardiovasc Diagn. 1988;15:192-202.

13. Swijnenburg RJ, Tanaka M, Vogel H, Baker J, Kofidis T, Gunawan F, et al. Embryonic stem cell immunogenicity increases upon differentiation after transplantation into ischemic myocardium. Circulation. 2005;112(9 Suppl):I166-72.

14. Muller-Ehmsen J, Whittaker P, Kloner RA, Dow JS, Sakoda T, Long TI, et al. Survival and development of neonatal rat cardiomyocytes transplanted into adult myocardium. J Mol Cell Cardiol. 2002;34:107-16.

15. Nussbaum J, Minami E, Laflamme MA, Virag JA, Ware CB, Masino A, et al. Transplantation of undifferentiated murine embryonic stem cells in the heart: teratoma formation and immune response. FASEB J. 2007;21: 1345-57.

16. Jain M, DerSimonian H, Brenner DA, Ngoy S, Teller P, Edge AS, et al. Cell therapy attenuates deleterious ventricular remodeling and improves cardiac performance after myocardial infarction. Circulation. 2001;103: 1920-7.

17. Weisel RD, Li RK, Mickle DA, Yau TM. Cell transplantation comes of age. J Thorac Cardiovasc Surg. 2001;121(5):835-6.

18. Bhatnagar SK, al-Yusuf AR. Left ventricular blood flow analysis in patients with and without a thrombus after first $\mathrm{Q}$ wave acute anterior myocardial infarction: two-dimensional Doppler echocardiographic study. Angiology. 1992;43(3 Pt 1):188-94.

19. Li RK, Mickle DA, Weisel RD, Zhang J, Mohabeer MK. In vivo survival and function of transplanted rat cardiomyocytes. Circ Res. 1996; 78:283-8.

20. Chen Y, Ke Q, Yang Y, Rana JS, Tang J, Morgan JP, Xiao YF. Cardiomyocytes overexpressing TNF-alpha attract migration of embryonic stem cells via activation of $\mathrm{p} 38$ and c-Jun amino-terminal kinase. FASEB J. 2003;17:2231-9.

21. Kurrelmeyer KM, Michael LH, Baumgarten G, Taffet GE, Peschon JJ, Sivasubramanian N, et al. Endogenous tumor necrosis factor protects the adult cardiac myocyte against ischemic-induced apoptosis in a murine model of acute myocardial infarction. Proc Natl Acad Sci U S A. 2000;97:5456-61.

22. Nakano M, Knowlton AA, Dibbs Z, Mann DL. Tumor necrosis factoralpha confers resistance to hypoxic injury in the adult mammalian cardiac myocyte. Circulation. 1998;97:1392-400.

23. Sivasubramanian N, Coker ML, Kurrelmeyer KM, MacLellan WR, DeMayo FJ, Spinale FG, et al. Left ventricular remodeling in transgenic mice with cardiac restricted overexpression of tumor necrosis factor. Circulation. 2001;104:826-31.

24. Gunja-Smith Z, Morales AR, Romanelli R, Woessner JF Jr. Remodeling of human myocardial collagen in idiopathic dilated cardiomyopathy. Role of metalloproteinases and pyridinoline cross-links. Am J Pathol. 1996;148:1639-48.

25. Yu Q, Horak K, Larson DF. Role of T lymphocytes in hypertension-induced cardiac extracellular matrix remodeling. Hypertension. 2006;48: 98-104.

26. Klug MG, Soonpaa MH, Koh GY, Field LJ. Genetically selected cardiomyocytes from differentiating embryonic stem cells form stable intracardiac grafts. J Clin Invest. 1996;98:216-24.

27. Soonpaa MH, Koh GY, Klug MG, Field LJ. Formation of nascent intercalated disks between grafted fetal cardiomyocytes and host myocardium. Science. 1994;264:98-101.

28. Cao F, Lin S, Xie X, Ray P, Patel M, Zhang X, et al. In vivo visualization of embryonic stem cell survival, proliferation, and migration after cardiac delivery. Circulation. 2006;113:1005-14. 
TABLE E1. Steady-state hemodynamic measurements by magnetic resonance imaging and pressure-volume loop

\begin{tabular}{|c|c|c|c|c|}
\hline & \multirow[b]{2}{*}{ Normal (no infarct) } & \multicolumn{3}{|c|}{ Infarct treatment groups } \\
\hline & & mESC & $\mathrm{mEF}$ & Normal saline \\
\hline \multicolumn{5}{|l|}{ Magnetic resonance imaging } \\
\hline LV ejection fraction $(\%)$ & $60.9 \pm 1.4$ & $40.2 \pm 2.0$ & $29.4 \pm 1.5$ & $26.4 \pm 1.8$ \\
\hline LV end-diastolic volume (mL) & $4.23 \pm 0.5$ & $4.53 \pm 0.6$ & $5.74 \pm 0.6$ & $8.32 \pm 0.7$ \\
\hline LV end-systolic volume (mL) & $1.63 \pm 0.2$ & $2.67 \pm 0.2$ & $4.09 \pm 0.3$ & $6.14 \pm 0.5$ \\
\hline \multicolumn{5}{|l|}{ Pressure-volume loop } \\
\hline Heart rate (beats/min) & $277.25 \pm 10.60$ & $302.75 \pm 26.93$ & $319.25 \pm 16.45$ & $260.00 \pm 22.51$ \\
\hline End-systolic volume $(\mu \mathrm{L})$ & $26.72 \pm 0.49$ & $21.65 \pm 4.82$ & $40.56 \pm 8.67$ & $45.82 \pm 8.89$ \\
\hline End-diastolic volume $(\mu \mathrm{L})$ & $29.29 \pm 0.47$ & $24.00 \pm 5.56$ & $43.15 \pm 9.21$ & $49.40 \pm 9.67$ \\
\hline End-systolic pressure $(\mathrm{mm} \mathrm{Hg})$ & $83.86 \pm 9.17^{*}$ & $85.92 \pm 5.91^{*}$ & $71.40 \pm 6.55$ & $49.84 \pm 5.35$ \\
\hline End-diastolic pressure $(\mathrm{mm} \mathrm{Hg})$ & $5.15 \pm 1.21$ & $19.14 \pm 7.01$ & $6.50 \pm 0.54$ & $8.21 \pm 1.64$ \\
\hline Arterial elastance $(\mathrm{mm} \mathrm{Hg} / \mu \mathrm{L})$ & $29.34 \pm 9.50$ & $37.50 \pm 11.56$ & $22.04 \pm 3.88$ & $10.62 \pm 3.35$ \\
\hline Maximum differential of pressure $(\mathrm{mm} \mathrm{Hg} / \mathrm{s})$ & $4100.50 \pm 412.10$ & $3867.50 \pm 751.61$ & $3108.75 \pm 293.20$ & $2157.00 \pm 358.83$ \\
\hline Minimum differential of pressure $(\mathrm{mm} \mathrm{Hg} / \mathrm{s})$ & $-2993.25 \pm 138.66^{*}$ & $-2779.75 \pm 144.06^{*}$ & $-2397.75 \pm 107.80$ & $-1578.50 \pm 304.29$ \\
\hline Tau (Weiss) & $15.01 \pm 1.32$ & $21.38 \pm 4.32$ & $15.13 \pm 1.16$ & $12.75 \pm 3.89$ \\
\hline Maximal power (mW) & $0.79 \pm 0.10$ & $0.78 \pm 0.07$ & $1.15 \pm 0.45$ & $0.84 \pm 0.27$ \\
\hline $\begin{array}{l}\text { Preload-adjusted maximal } \\
\text { power }\left(\mathrm{mW} / \mu \mathrm{L}^{2}\right)\end{array}$ & $9.21 \pm 1.19$ & $18.88 \pm 6.13^{*}$ & $5.87 \pm 0.76$ & $3.69 \pm 1.25$ \\
\hline
\end{tabular}

Values are mean \pm SE. $m E S C$, Mouse embryonic stem cell treatment; $m E F$, mouse embryonic fibroblast treatment; $L V$, left ventricle. ${ }^{*} P<.05$ versus normal saline group by analysis of variance. 